PROCEEDINGS OF THE

AMERICAN MATHEMATICAL SOCIETY

Volume 133, Number 6, Pages 1621-1628

S 0002-9939(05)07736-1

Article electronically published on January 14, 2005

\title{
POROSITY AND DIFFERENTIABILITY IN SMOOTH BANACH SPACES
}

\author{
PANDO GR. GEORGIEV \\ (Communicated by Jonathan M. Borwein) \\ Dedicated to Professor Petar Kenderov on the occasion of his 60th anniversary

\begin{abstract}
We improve a result of Preiss, Phelps and Namioka, showing that every submonotone mapping in a Gateaux smooth Banach space is singlevalued on the complement of a $\sigma$-cone porous subset. If a Banach space $E$ has a uniformly $\beta$-differentiable Lipschitz bump function (with respect to some bornology $\beta$ ), then we show with a much simpler argument (localization of $\delta$-minimum of a perturbed function) that every continuous convex function on
\end{abstract} \\ $E$ is $\beta$-differentiable on the complement of a $\sigma$-uniformly porous set.
}

\section{INTRODUCTION}

A result of Preiss-Phelps-Namioka [9] states that every monotone mapping $T$ : $E \rightarrow 2^{E^{*}}$, where $E$ is a Banach space with an equivalent Gateaux differentiable norm, is single-valued on a residual subset $R_{T}$ of $\operatorname{int} D(T)$, where $D(T)=\{x \in$ $E: T(x) \neq \emptyset\}$ (i.e. the complement of $R_{T}$ is of first Baire category). In particular, $E$ is weak Asplund, i.e. every continuous convex function defined on it is Gateaux differentiable on a residual subset. N. Ribarska 11] generalized this result in the sense that she additionally showed that the dual space of $E$ with the weak* topology is fragmentable (see the definition below), from which the results in [9] straightforwardly follow. We note that there are weak Asplund spaces whose duals are not weak* fragmentable (see Kenderov et al. [7]).

Here we extend the above-mentioned result of Preiss-Phelps-Namioka in two directions: the first one is that we work with submonotone mappings $T$ (see the definition below) instead of monotone mappings, and the second is that the set $R_{T}$ is not only residual, but is a complement of a $\sigma$-cone porous set (and therefore is $\sigma$-porous). In the proof we use ideas from Zajicek's paper [13] and results of N. Ribarska [10], [11].

Another result in the present paper states that in the case when the space possesses a uniformly $\beta$-differentiable Lipschitz bump function with respect to some bornology $\beta$, then every continuous convex function is $\beta$-differentiable on a subset whose complement is $\sigma$-uniformly porous. The proof of this result uses a simple localization of a $\delta$-minimum of a lower semicontinuous bounded below function. In the case when the bornology consists of all finite subsets, then the result concerns

Received by the editors July 31, 2002.

2000 Mathematics Subject Classification. Primary 49J53; Secondary $49 J 50$.

Key words and phrases. Porous set, submonotone mappings, differentiability.

(c)2005 American Mathematical Society 1621

Reverts to public domain 28 years from publication 
Gateaux differentiability and is known (see Zajicek [14], where a much more general result is proved).

\section{Porous sets}

We recall the following definitions (see [13). Let $P$ be a metric space, $(E,\|\|$.$) a$ Banach space with a dual $\left(E^{*},\|\cdot\|\right), M \subset P, x \in P$, and $R>0$. Denote by $B(x ; R)$ the open ball with center $x$ and radius $R$ and put

$$
\gamma(x, R, M)=\sup \{r>0: \exists z \in P: B(z ; r) \subset B(x ; R) \backslash M\} .
$$

The number $p(x, M)=\lim \sup _{R \rightarrow 0+} \gamma(x, R, M) R^{-1}$ is called a porosity constant of $M$ at $x$. If $p(x, M)>0$, then $M$ is called porous at $x$. $M$ is called porous if it is porous at every one of its points. We will call $M$ uniformly porous if $\inf _{x \in M} p(x, M)>0 . \quad M$ is called $\sigma$-porous (resp. $\sigma$-uniformly porous) if it can be presented as a union of countably many porous (resp. uniformly porous) sets.

Every $\sigma$-porous set is of first Baire category and, in finite-dimensional spaces, has a measure zero. But there are simple examples of sets of first category, which have nonzero measure.

For $v \in E$ and $c>0$ define the cone

$$
A(v, c)=\{x \in E: x=\lambda v+w, \lambda>0,\|w\|<c \lambda\}=\bigcup_{\lambda>0} \lambda B(v ; c) .
$$

A subset $M \subset E$ is called cone porous at $x \in M$ if there exist $R>0, v \in E,\|v\|=1$ and $c \in(0,1)$ such that

$$
M \cap B(x ; R) \cap(x+A(v, c))=\emptyset .
$$

$M$ is called cone porous if it is cone porous at every one of its points. $M$ is called $\sigma$-cone porous if it is a union of countably many cone porous sets.

It is easy to see that every $\sigma$-cone porous set is $\sigma$-porous.

In the proof of the next theorem we need the following.

Proposition 2.1 (13, Proposition 1]). Let $E$ be a Banach space and let the set $M \subset E$ be not $\sigma$-cone porous. Then there exists $\emptyset \neq N \subset M$ such that $N$ is not cone porous at any point of $N$.

\section{Fragmentable spaces}

We will recall the following definition (see [10]).

A well-ordered family $\mathcal{U}=\left\{U_{\xi}: 0 \leq \xi<\xi_{0}\right\}$ of subsets of a topological space $X$ is called a relatively open partition of $X$ if

(i) $U_{0}=\emptyset$;

(ii) $U_{\xi}$ is contained in $X \backslash\left(\bigcup_{\eta<\xi} U_{\eta}\right)$ and is relatively open in it for every $\xi, 0<\xi<\xi_{0}$

(iii) $X=\bigcup_{\xi<\xi_{0}} U_{\xi}$.

A family $\mathcal{U}$ of sets of the topological space $X$ is called a $\sigma$-relatively open partition of $X$ if $\mathcal{U}=\bigcup_{n=1}^{\infty} \mathcal{U}^{n}$, where $\mathcal{U}^{n}, n=1,2, \ldots$, are relatively open partitions of $X$. $\mathcal{U}$ separates the points of $X$ if for every two different points $x, y \in X$ there exists $n$ such that $x$ and $y$ belong to different elements of the partition $\mathcal{U}^{n}$.

A topological space $X$ is called fragmentable if there exists a metric $\rho$ on it such that every nonempty subset of $X$ has nonempty relatively open subsets with arbitrarily small $\rho$-diameter. 
A result of N. Ribarska [10] states that a topological space $X$ is fragmentable if and only if it possesses a $\sigma$-relatively open partition which separates the points of $X$. In the proof of Theorem 5.1 we use this result as well as the formula for the fragmentable metric, given in [10].

\section{Submonotone mappings}

Definition 4.1 ([5]). A mapping $T: E \rightarrow 2^{E^{*}}$ is called submonotone at $x_{0} \in E$ provided

$$
\liminf _{\substack{x_{0} \neq x \rightarrow x_{0} \\ y \in T(x), y_{0} \in T\left(x_{0}\right)}} \frac{\left\langle x-x_{0}, y-y_{0}\right\rangle}{\left\|x-x_{0}\right\|} \geq 0
$$

for every $e \in E,\|e\|=1$, where $x \rightarrow_{e} x_{0}$ denotes that $x$ converges to $x_{0}$ in direction $e$ (i.e. $x \rightarrow x_{0}$ and $\frac{x-x_{0}}{\left\|x-x_{0}\right\|} \rightarrow e$ ).

We need the following characterization of submonotone mappings (see [5, Theorem 3.1]). First denote

$$
U\left(x_{0}, e, \delta\right)=\left\{x \in E: x \neq x_{0},\left\|x-x_{0}\right\|<\delta,\left\|\frac{x-x_{0}}{\left\|x-x_{0}\right\|}-e\right\|<\delta\right\}
$$

and recall that a mapping $T: E \rightarrow 2^{E^{*}}$ is directionally locally bounded at $x_{0} \in E$ if for every $e \in E,\|e\|=1$, there exists $\delta>0$ such that $T\left(U\left(x_{0}, e, \delta\right)\right)$ is bounded.

Lemma 4.2 ([5, Theorem 3.1]). Let the mapping $T: E \rightarrow 2^{E^{*}}$ be directionally locally bounded at $x_{0} \in E$. Then $T$ is submonotone at $x_{0}$ if and only if for every $e \in E,\|e\|=1$, and every $\varepsilon>0$ there exists $\delta>0$ such that

$$
\inf \langle e, T(x)\rangle \geq \sup \left\langle e, T\left(x_{0}\right)\right\rangle-\varepsilon \text { for every } x \in U\left(x_{0}, e, \delta\right) \text {. }
$$

\section{MAin RESUlt}

The next theorem contains the first main result in the paper.

Theorem 5.1. Let $E$ be a Gateaux smooth Banach space and let $T: E \rightarrow 2^{E^{*}}$ be a locally bounded submonotone operator with an arbitrary domain $D(T)=\{x$ : $T(x) \neq \emptyset\}$. Then there exists a $\sigma$-cone porous set $A \subset D(T)$ such that $T$ is singlevalued at every point of $D(T) \backslash A$.

Proof. Here we combine ideas from the proof of [13, Theorem 1] (where the statement of the theorem is proved in the case when $E$ is an Asplund space and $T$ is a monotone operator) and ideas from [10], [11].

Further in the proof we adopt the notations from the paper of N. Ribarska [11]. From the proof of Theorem 1.1 in [11] it follows that there exists a separating $\sigma$ relatively open partition $\mathcal{U}=\bigcup_{n=1}^{\infty} \mathcal{U}^{n}$ of the dual unit ball with the weak ${ }^{*}$-topology $\left(B^{*}, w^{*}\right)$ such that every element $U_{\xi}^{n}$ of $\mathcal{U}^{n}=\left\{U_{\xi}^{n}: 0 \leq \xi \leq \xi_{n}\right\}$ has a relatively open partition $\mathcal{U}_{\xi}^{n}=\left\{U_{\xi \eta}^{n}: 0 \leq \eta<\eta_{\xi}^{n}\right\}$, where $U_{\xi \eta}^{n}$ is a slice of $U_{\xi}^{n} \backslash \bigcup_{\eta<\xi} U_{\xi \eta}^{n}$, $U_{\xi 0}^{n}=\emptyset$ and $\mathcal{U}^{n+1}=\bigcup\left\{\mathcal{U}_{\xi}^{n}: 0 \leq \xi \leq \xi_{n}\right\}$ as $U_{\xi \eta}^{n}: 0 \leq \xi<\xi_{n}, 0 \leq \eta<\eta_{\xi}^{n}$ are ordered lexicographically. 
From another result of N. Ribarska [10] it follows that $\left(B^{*}, w^{*}\right)$ is fragmentable and the metric which fragments it is

$$
\rho(x, y)= \begin{cases}0 & \text { if } x=y, \\ \left(\min \left\{n: \mathcal{U}^{n} \operatorname{separates} x \text { and } y\right\}\right)^{-1} & \text { otherwise }\end{cases}
$$

and $\rho$ - $\operatorname{diam}\left(U_{\xi}^{n}\right)<1 / n$ for every $n \in \mathbb{N}$.

Assume that the statement of the theorem is not valid: the set

$$
A:=\{x \in D(T): T \text { is not single-valued at } x\}
$$

is not $\sigma$-cone porous. Since

$$
A=\bigcup_{n=1}^{\infty} A_{n}
$$

where

$$
A_{n}=\{x \in D(T): \rho-\operatorname{diam} T(x)>1 / n\},
$$

we can choose a positive integer $n$ such that $A_{n}$ is not $\sigma$-cone porous. By Proposition 2.1 there exists a set $\emptyset \neq N \subset A_{n}$ which is not cone porous at any of its points. Choose $x^{\prime} \in N$. Since $T$ is locally bounded, there exists $r$ such that $T\left(B\left(x^{\prime} ; r\right)\right)$ is bounded. Putting $H=N \cap B\left(x^{\prime} ; r\right)$, we see that $\emptyset \neq H$ is not cone porous at any point of $H$ and $T(H)$ is a bounded subset of $X^{*}$, so there exists $K>0$ such that $\left\|y^{*}\right\|<K$ for every $y^{*} \in T(H)$.

Without loss of generality we may assume that $K=1$, i.e. $T(H)$ is a subset of the closed unit ball $B^{*}$ of $E^{*}$.

Let $\gamma_{1}=\min \left\{\xi: U_{\xi}^{1} \cap T(H) \neq \emptyset\right\}$ and let $S_{1}:=U_{\gamma_{1}}^{1} \cap T(H)$. By construction (see the proof of Theorem 1.1 in [11]), $S_{1}$ is a slice (Lebesgue's set) of $T(H)$ determined by an element $e_{\gamma_{1}} \in E,\left\|e_{\gamma_{1}}\right\|=1$, and by a positive number $\alpha_{\gamma_{1}}$ :

$$
S_{1}=\left\{y \in T(H):\left\langle e_{\gamma_{1}}, y\right\rangle>\sup \left\{\left\langle e_{\gamma_{1}}, z\right\rangle-\alpha_{\gamma_{1}}: z \in T(H)\right\}\right\} .
$$

We have that $\rho$-diam of $S_{1}$ is less than 1 .

We choose $x_{1} \in H$ such that $S_{1} \cap T\left(x_{1}\right) \neq \emptyset$ and let $y \in S_{1} \cap T\left(x_{1}\right)$. Since the mapping $T$ is submonotone, by Lemma 4.2 there exists $\delta_{1}>0$ such that $T\left(U\left(x_{1}, e_{\gamma_{1}}, \delta_{1}\right)\right) \subset S_{1}$. Since $H$ is not cone porous at $x_{1}$, the set $H_{1}:=H \cap$ $U\left(x_{1}, e_{\gamma_{1}}, \delta_{1}\right)$ is nonempty and therefore $T\left(H_{1}\right) \subset S_{1}$ and $\rho$-diam $T\left(H_{1}\right)<1$.

Let $\gamma_{2}=\min \left\{\xi: U_{\xi}^{2} \cap T\left(H_{1}\right) \neq \emptyset\right\}$ and let $S_{2}:=U_{\gamma_{2}}^{2} \cap T\left(H_{1}\right)$. By construction, $S_{2}$ is a slice (Lebesgue's set) of $T\left(H_{1}\right)$ determined by an element $e_{\gamma_{2}} \in E,\left\|e_{\gamma_{2}}\right\|=1$, and by a positive number $\alpha_{\gamma_{2}}$ :

$$
S_{2}=\left\{y \in T\left(H_{1}\right):\left\langle e_{\gamma_{2}}, y\right\rangle>\sup \left\{\left\langle e_{\gamma_{2}}, z\right\rangle-\alpha_{\gamma_{2}}: z \in T\left(H_{1}\right)\right\}\right\} .
$$

We have that $\rho$-diam of $S_{2}$ is less than $1 / 2$ and so on. In the $n$-th step we obtain a slice $S_{n}$ such that $\rho$-diam of $S_{n}$ is less than $1 / n$, and a set $H_{n} \subset H$ for which $T\left(H_{n}\right) \subset S_{n}$, a contradiction with (5.1). 


\section{UNIFORM POROSITY AND DIFFERENTIABILITY}

We shall recall that a function $b: E \rightarrow \mathbb{R}$, defined in an arbitrary Banach space $E$, is called a bump function if there exists a bounded subset supp $b \subset E$, such that $b(x)=0$ for every $x \notin \operatorname{supp} b$.

The following proposition, which gives a simple localization of the $\delta$-minimum of a perturbed function, can be considered as a prototype of the Deville-GodefroyZizler variational principle [3], concerning the density part of the latter. Indeed, this density part follows easily by iteratively applying this localization with $\delta_{n}$ converging to zero. It turns out that such a simple $\delta$-localization is enough to obtain results about generic differentiability (see [6]) and even more results about differentiability on the complement of a $\sigma$-uniformly porous set.

Proposition 6.1 ([6]). Let $b$ be a bump function, such that supp $b \subset B(0 ; 1)$, $b(0)=1$ and $0 \leq b(x) \leq 1$ for every $x \in E$. Let $f: E \rightarrow \mathbb{R} \cup\{+\infty\}$ be a function, bounded from below and such that $D(f)=\{x \in E: f(x)<+\infty\} \neq \emptyset$. Let $\varepsilon>0, \lambda>0$ be given. Suppose that $y_{0} \in X$ satisfies the condition

$$
f\left(y_{0}\right)<\inf _{E} f+\varepsilon .
$$

Then for every $\delta>0$ there exists a point $x_{0} \in E$, such that:

(a) $\quad f\left(x_{0}\right)-\varepsilon b\left(\frac{x_{0}-y_{0}}{\lambda}\right)<f(x)-\varepsilon b\left(\frac{x-y_{0}}{\lambda}\right)+\delta$ for every $x \in E$;

(b) $\frac{x_{0}-y_{0}}{\lambda} \in \operatorname{supp} b$;

(c) $\quad\left\|x_{0}-y_{0}\right\|<\lambda$.

Proof. Denote

Then

$$
h(x)=f(x)-\varepsilon b\left(\frac{x-y_{0}}{\lambda}\right) .
$$

$$
h\left(y_{0}\right)=f\left(y_{0}\right)-\varepsilon<\inf _{E} f
$$

Let

$$
\delta_{1}=\inf _{E} f-h\left(y_{0}\right)
$$

There exists a point $x_{0} \in E$ such that

$$
h\left(x_{0}\right)<\inf _{E} h+\min \left\{\delta, \delta_{1}\right\},
$$

which proves (a).

Assume that $\frac{x_{0}-y_{0}}{\lambda} \notin \operatorname{supp} b$. Then

$$
\delta_{1}>h\left(x_{0}\right)-h\left(y_{0}\right)=f\left(x_{0}\right)-\inf _{E} f+\delta_{1} \geq \delta_{1},
$$

which is a contradiction. So (b) is fulfilled. It is clear that (c) follows immediately from (b).

We will recall the following notion of 'bornology'. For motivation and applications of this notion see [1], 2].

The bornology $\beta$ on $E$ is a family of closed, bounded and symmetric (with respect to 0 ) subsets of $E$, whose union is $E$, which is closed with respect to multiplication with real numbers and has the property that the union of every two members of $\beta$ is contained in $\beta$. We shall denote by $E_{\beta}^{*}$ the dual space of $E$ furnished with the topology of the uniform convergence on the sets from $\beta$. The most important 
bornologies are those consisting of all (symmetric) bounded sets (Fréchet bornology), of all weakly compact subsets (weak Hadamard bornology), of all compact sets (Hadamard bornology), and of all finite sets (Gateaux bornology).

For a given function $f: E \rightarrow \mathbb{R} \cup\{+\infty\}$ we say that $f$ is $\beta$-differentiable at $x$ and has $\beta$-derivative $\nabla^{\beta} f(x)$ if $f(x)$ is finite and

$$
\frac{f(x+t h)-f(x)}{t}-\left\langle\nabla^{\beta} f(x), h\right\rangle \rightarrow 0
$$

as $t \rightarrow 0$ uniformly on $h \in H$ for every $H \in \beta$. If $f$ is a bump function and, in addition, the convergence in 6.1 is uniform also with respect to $x \in E$, then $f$ is called uniformly $\beta$-differentiable.

In what follows we need the following proposition, whose proof is analogous to that of [8, Proposition 1.23].

Proposition 6.2. A continuous convex function $f: E \rightarrow \mathbb{R}$ is $\beta$-differentiable at $x$ if and only if for every $\varepsilon>0$ and every $H \in \beta$ there exists $t>0$ such that

$$
\frac{f(x+t h)+f(x-t h)-2 f(x)}{t}<\varepsilon \text { for every } h \in H .
$$

Theorem 6.3. Assume that the Banach space $E$ has a uniformly $\beta$-differentiable Lipschitz bump function $\tilde{b}$. Then any convex continuous function $f$ defined on an open subset $U$ of $E$ is $\beta$-differentiable on the complement of a $\sigma$-uniformly porous subset of $U$.

Proof. Without loss of generality we may assume that $\tilde{b}(0) \neq 0$. Define a new bump function $b: E \rightarrow \mathbb{R}$ as $b(x):=\tau(\tilde{b}(\mu x))$, where $\mu=\sup _{s \in \operatorname{supp} \tilde{b}}\|s\|$ and $\tau: \mathbb{R} \rightarrow \mathbb{R}$ is a nonnegative continuously differentiable bump function such that $0 \notin \operatorname{supp} \tau$ and $\tau(\tilde{b}(0))=\max _{t \in \mathbb{R}} \tau(t)=1$. It is easy to see, using the Lipschitz assumption on $\tilde{b}$, that $b$ is uniformly $\beta$-differentiable and satisfies the assumptions of Proposition 6.1

Consider the set

$$
\begin{aligned}
X_{n, m}=\{ & x \in U: \forall H \in B_{n, m} \quad \exists t \in(0,1 / m]: \\
& \left.\frac{f(x+t h)+f(x-t h)-2 f(x)}{t}<\frac{8}{n} \quad \forall h \in H\right\},
\end{aligned}
$$

where

$$
B_{n, m}=\left\{H \in \beta:-b\left(x+\frac{1}{m} h\right)-b\left(x-\frac{1}{m} h\right)+2 b(x)<\frac{1}{m n} \quad \forall h \in H, \forall x \in E\right\} .
$$

Since $f$ is convex and continuous, it is locally Lipschitz. A set is $\sigma$-uniformly porous if it is locally $\sigma$-uniformly porous [13] (i.e. if it is $\sigma$-uniformly porous in a neighborhood of any of its points). So, without loss of generality we may assume that $f$ is Lipschitz on $U$ with a Lipschitz constant 1 .

We shall prove that $E \backslash X_{n, m}$ is uniformly porous. Assume the contrary. Then, by definition, for $\alpha \in(0,1 /(2 m n))$ we have

$$
\exists x_{0} \in U: \exists R \in(0, \alpha): B(y ; \alpha R) \cap\left(E \backslash X_{n, m}\right) \neq \emptyset \quad \forall y \in B\left(x_{0} ; r R(1-\alpha)\right) .
$$

From the Lipschitz property of $f$ we have

$$
f\left(x_{0}\right)-f(x) \leq\left\|x-x_{0}\right\|<R \quad \forall x \in B\left(x_{0} ; R\right) .
$$


From Proposition 6.1 applied for the function $-f$ with $\varepsilon=R=2 \lambda$ and $\delta=$ $R /(2 m n)$, there exists $y \in B\left(x_{0} ; R / 2\right)$ such that

$$
-f(y)-\varepsilon b\left(\frac{y-x_{0}}{\lambda}\right) \leq-f(x)-\varepsilon b\left(\frac{x-x_{0}}{\lambda}\right)+\delta \quad \forall x \in B\left(x_{0} ; R\right) .
$$

By 6.2 there exists $z \in B(y ; \alpha R) \cap\left(E \backslash X_{n, m}\right)$, so by definition of $X_{n, m}$, there exists $H \in B_{n, m}$ such that for $t=R / 2 m$ there is $h \in H$ for which we can write

$$
\begin{aligned}
\frac{8}{n} & \leq \frac{f(z+t h)+f(z-t h)-2 f(z)}{t} \\
& \leq \frac{f(y+t h)+f(y-t h)-2 f(y)}{t}+4 / n \\
& \leq-\frac{\varepsilon}{t} b\left(\frac{y+t h-x_{0}}{\lambda}\right)-\frac{\varepsilon}{t} b\left(\frac{y-t h-x_{0}}{\lambda}\right)+2 \frac{\varepsilon}{t} b\left(\frac{y-x_{0}}{\lambda}\right)+\frac{4}{n}+2 \frac{\delta}{t} \\
& =2 m\left[-b\left(\frac{y-x_{0}}{\lambda}+\frac{1}{m} h\right)-b\left(\frac{y-x_{0}}{\lambda}-\frac{1}{m} h\right)+2 b\left(\frac{y-x_{0}}{\lambda}\right)\right]+\frac{4}{n}+\frac{4 m}{R} \delta \\
& <\frac{2}{n}+\frac{4}{n}+\frac{2}{n}=\frac{8}{n},
\end{aligned}
$$

which is a contradiction. Therefore the set $E \backslash X_{n, m}$ is uniformly porous.

Since $b$ is $\beta$-differentiable, we have $\bigcup_{m=1}^{\infty} B_{n, m}=\beta$ for every $n \geq 1$, therefore by Proposition 6.2, $f$ is $\beta$-differentiable on $\bigcap_{n, m=1}^{\infty} X_{n, m}$.

Remark 6.4. In the case of Gateaux bornology, the above theorem is a particular case of a more general result of L. Zajicek [14], concerning functions with directional derivatives. In this case the assumption that the space admits a uniformly Gateaux differentiable Lipschitz bump function is equivalent to the assumptions that the space admits an equivalent uniformly Gateaux differentiable norm (see [12]).

\section{REFERENCES}

1. J.M. Borwein and Q. Zhu, Viscosity solutions and viscosity subderivatives in smooth Banach spaces with applications to metric regularity, SIAM J. Control Optim. 34 (1996), no. 5, 15681591. MR 1404847 (97g:49037)

2. J.M. Borwein, J.S. Treiman and Q.J. Zhu, Partially smooth variational principles and applications, Nonlinear Anal. 35 (1999), no. 8, Ser. B: Real World Applications, 1031-1059. MR1707806 (2000j:49028)

3. R. Deville, G. Godefroy and V. Zizler, A smooth variational principle with applications to Hamilton-Jacobi equations in infinite dimensions, J. Funct. Anal. 111 (1993), 197-212. MR 1200641 (94b:49010)

4. I. Ekeland and G. Lebourg, Generic Fréchet differentiabili ty and perturbed optimization problems in Banach spaces, Trans. Amer. Math. Soc. 224 (1979), 193-216. MR0431253 $(55: 4254)$

5. P.G. Georgiev, Submonotone mappings in Banach spaces and applications, Set-Valued Anal. 5 (1997), no. 1, 1-35. MR1451845 (98d:49021)

6. P.G. Georgiev and N.P. Zlateva, Generic Gateaux differentiability via smooth perturbations, Bull. Austral. Math. Soc. 56 (1997), 421-428. MF 1490659 (98i:46039)

7. P.S. Kenderov, W.B. Moors and S. Sciffer, A weak Asplund space whose dual is not weak* fragmentable, Proc. Amer. Math. Soc. 129 (2001), 3741-3747. MR1860511 (2002h:54014)

8. R.R. Phelps, Convex Functions, Monotone Operators and Differentiability, Lecture Notes in Mathematics, No. 1364. MR0984602 (90g:46063)

9. D. Preiss, R.R. Phelps and I.Namioka, Smooth Banach spaces, weak Asplund spaces and monotone or usco mappings, Israel J. Math. 72 (1990), 257-279. MR1120220 (92h:46021)

10. N.K. Ribarska, Internal characterization of fragmentable spaces, Mathematika 34 (1987), 243-257. MR0933503 (89e:54063) 
11. N.K. Ribarska, The dual of a Gateaux smooth Banach space is weak star fragmentable, Proc. Amer. Math. Soc. 114 (1992), 1003-1008. MR.1101992 (92g:46020)

12. W.K. Tang, Uniformly Differentiable Bump Functions, Arch. Math. 68 (1997), 55-59. MR 1421846 (97j:46010)

13. L. Zajicek, Smallnesss of sets of nondifferentiability of convex functions in non-separable Banach spaces, Czech. Math. J. 41 (116) (1991), 288-296. MF.1105445 (92d:46112)

14. L. Zajicek, On differentiability properties of Lipschitz functions on a Banach space with uniformly Gateaux differentiable bump function, Comment. Math. Univ. Carolinae 38 (1997), 329-336. MR1455499 (99b:46066)

Department of Mathematics and Informatics, Sofia University "St. Kl. Ohridski", 5 James Bourchier Boulevard, 1126 Sofia, Bulgaria

Current address: Electrical \& Computer Engineering and Computer Science Department, University of Cincinnati, ML 0030, Cincinnati, Ohio 45220

E-mail address: pgeorgie@ececs.uc.edu 